\title{
SRPX and HMCN1 regulate cancer-associated fibroblasts to promote the invasiveness of ovarian carcinoma
}

\author{
CHAO LIEN LIU ${ }^{1}$, HUNG WEI PAN ${ }^{2}$, PAO LING TORNG ${ }^{3}$, MING HUEI FAN ${ }^{1}$ and TSUI LIEN MAO ${ }^{4}$ \\ ${ }^{1}$ School of Medical Laboratory Science and Biotechnology, College of Medical Science and Technology, \\ Taipei Medical University, Taipei 11031; ${ }^{2}$ Department of Medical Education and Research, \\ Kaohsiung Veterans General Hospital, Kaohsiung 81362; ${ }^{3}$ Department of Obstetrics and Gynecology, \\ National Taiwan University Hospital, Hsin-Chu Branch, Hsinchu 30059; ${ }^{4}$ Department of Pathology, \\ College of Medicine, National Taiwan University, Taipei 10002, Taiwan, R.O.C.
}

Received February 9, 2019; Accepted August 22, 2019

DOI: 10.3892/or.2019.7379

\begin{abstract}
Cancer-associated fibroblasts (CAFs) are known to be essential in cancer initiation and development. However, the role of CAFs in promoting ovarian cancer (OC) invasion remains to be fully elucidated. To address this in the present study, 49 clinical OC specimens were used to evaluate the roles of CAFs in promoting ovarian tumor migration and invasion and disease progression. It was found that the sushi repeat-containing protein, $\mathrm{X}$-linked $(S R P X)$ and hemicentin 1 (HMCN1) genes were significantly upregulated in CAFs from high-grade serous carcinoma (HGSC) and clear cell carcinoma (CCC) samples, the two major histological types of $\mathrm{OC}$ with frequently poor patient survival rates. The short hairpin (sh)RNA-mediated silencing of SRPX and HMCNI in fibroblasts significantly suppressed the Transwell invasive activities of OC cells. Further experiments showed that SRPX and HMCN1 regulated the invasiveness of OC via the Ras homology family member A (RhoA) signaling pathway in fibroblasts. Therefore, the findings of the present study suggest that targeting the CAF genes, SRPX and HMCN1, can inhibit OC migration and invasion. These data highlight the importance of CAF-OC crosstalk signaling in cancer invasion and demonstrate the potential for improved efficacy of $\mathrm{OC}$ treatment by targeting CAF-SRPX/HMCN1.
\end{abstract}

\section{Introduction}

Ovarian carcinoma (OC) consists of five main, distinct histomorphological types: High-grade serous carcinoma (HGSC),

Correspondence to: Dr Tsui Lien Mao, Department of Pathology, College of Medicine, National Taiwan University, 7 Chung-San South Road, Taipei 10002, Taiwan R.O.C.

E-mail: tlmao@ntu.edu.tw

Key words: cancer-associated fibroblasts, ovarian cancer, sushi repeat-containing protein, X-linked, hemicentin 1, Ras homology family member A low-grade serous carcinoma, clear cell carcinoma (CCC), endometrioid carcinoma and mucinous carcinoma $(1,2)$. Among these, HGSC is the most common type, accounting for $>70 \%$ of cases of epithelial OC and portends the poorest prognosis (3). Surgical debulking and platinum-based chemotherapy have been used as first-line therapies for patients with OC for $>30$ years. Although the initial response is often positive, the disease usually recurs within 2-5 years as the tumor cells develop chemoresistance $(4,5)$. Numerous studies searching for prognostic indicators, via genomics, proteomics and metabolomics (6), and treatment approaches for OC have been reported, however, treatment success remains limited. Therefore, novel approaches for investigating factors related to the aggressiveness and recurrence of OC may provide potential targets for effective therapies.

Fibroblasts within the tumor stroma, known as cancer-associated fibroblasts (CAFs), are major cells in the tumor microenvironment (TME) and serve crucial roles in tumor growth, progression, metastasis, angiogenesis and immune responses (7-9). CAFs were originally described as a heterogeneous subpopulation of fibroblasts, which are activated by tumor cells and display specific markers that can be used as prognostic biomarkers $(10,11)$. The crosstalk between CAFs and other cells in the TME involves a wide range of factors, including chemokines [e.g., stromal cell-derived factor-1 $\alpha$ (12)], cytokines [(e.g., interleukin (IL)-6 (13), IL-8 and IL-1 $\beta$ (9)], exosomes [(e.g., cluster of differentiation $9^{+}$ exosomes (14) and growth factors (e.g., hepatocyte growth factor and transforming growth factor- $\beta$ ) (15)], which affect cancer cells and facilitate cancer-promoting processes (16). In addition, upon their activation, CAFs produce several mesenchyme-specific proteins, such as fibroblast-specific protein 1 (also referred to as S100A4) (17), a fibroblast activation protein $(18,19)$, vimentin and $\alpha$-smooth muscle actin, a prototypical marker of myofibroblasts. However, it remains unclear how cancer cells reprogram quiescent fibroblasts to become CAFs.

Reports on OC indicate that ovarian CAFs upregulate the lipoma-preferred partner $(L P P)$ gene in microvascular endothelial cells (MECs), and the expression levels of LPP in intratumoral MECs are correlated with survival rate and 
chemoresistance in patients with OC (20). By contrast, an in vivo imaging system showed that CAFs contribute to vascular remodeling and tumor angiogenesis in OC (21); furthermore, increasing evidence shows that mutations of CAF genes are crucial for cancer metastasis and growth. For example, certain changes in the DNA copy number of clustered CAFs may contribute to metastasis of breast cancer cells (22). Despite an increasing number of studies on CAFs, the majority have focused on cell line-based or animal models. OC is a heterogeneous group comprising different subtypes; how CAFs within different histological types of OC interact with cancer cells in the TME has not been investigated in detail.

The present study investigated the characteristics of fibroblasts isolated from 49 patients with various types of ovarian tumors and identified factors associated with cancer aggressiveness. The results revealed that the CAFs from patients with HGSC and CCC predominantly exhibited high invasion-promoting activities. The gene expression profiles of CAFs were elucidated using complementary DNA microarray analysis. Among these, SRPX and HMCN1, two genes with relatively high invasion-promoting activities, were upregulated in human HGSC CAFs. Additionally, the knockdown of SRPX and/or HMCN1 in WS1 fibroblasts significantly suppressed the migratory and invasive activities of OC cells, whereas the overexpression of SRPX in WS1 fibroblasts significantly increased in vitro OC cell invasive ability. Investigation of the mechanism underlying the effects of SRPX and HMCN1 knockdown in CAFs revealed that $S R P X$ and/or HMCN1 induced OC cell migration/invasion via activation of the Ras homology family member A (RhoA)/Rho kinase (ROCK)/myosin II regulatory light chain (MLC) signaling pathway. These results demonstrate that the importance of the TME and the significance of $\mathrm{CAFs}$ in promoting tumor invasion and aggressiveness in $\mathrm{OC}$ should not be underestimated.

\section{Materials and methods}

Isolation of ovarian CAFs and normal fibroblasts (NOFs). A total of 49 ovarian tumors of the major histological types (serous, endometrioid, clear cell and mucinous), which were diagnosed (reviewed by TLM) at the National Taiwan University Hospital during 2009-2011 and had been subjected to successful CAF isolation and Transwell invasion assay, were included for the study. The tumors were resected from patients aged 28-86 years (mean age, 50 years). Tissue samples were minced with a scalpel into $1-\mathrm{mm}^{3}$ pieces and digested with collagenase A $(1 \mathrm{mg} / \mathrm{ml})$ at $37^{\circ} \mathrm{C}$ for $40 \mathrm{~min}$. The cells were then seeded in a flask and cultured in Dulbecco's modified Eagle's medium (DMEM; Thermo Fisher Scientific, Inc.) for several days until confluent. For tumor samples, the tumor cells were isolated using the Ep-CAM antibody (Dynal); the negative portion containing the CAFs was harvested and grown in DMEM. The use of tissue samples was approved by the Ethics Committee and Review Board of National Taiwan University Hospital (IRB approval no. 200812141R). All patients signed written informed consent prior to the collection of tissues.

Cell culture. The TOV21G OC cell line and the WS1 skin fibroblast cell line (purchased from American Type Culture Collection), were cultured in DMEM supplemented with
$10 \% \mathrm{FBS}, 10 \mathrm{U} / \mathrm{ml}$ penicillin and $10 \mu \mathrm{g} / \mathrm{ml}$ streptomycin (Thermo Fisher Scientific, Inc.) at $37^{\circ} \mathrm{C}$ in an atmosphere containing $5 \% \mathrm{CO}_{2}$.

In vitro wound healing assay. To determine the ability of cells to migrate, a scratch wound-migration assay was performed. The TOV21G and WS1 cells (1:1) were seeded in six-well culture plates. The cells were grown to a confluent monolayer and then scraped with a 200- $\mu$ l pipette tip to generate scratch wounds. Migration from the edge of the injured monolayer was quantified by measuring the distance between the wound edges. Images were acquired using an Olympus microscope (BX50; Olympus Corporation) under a magnification of x200. The experiments were performed in triplicate.

In vitro Transwell invasion assay. Transwell chambers (Corning, New York, NY, USA) supplemented with growth factor (BD Biosciences, San Jose, CA, USA) were used for the invasion assays. Matrigel ( $15 \mu \mathrm{l}$ of $2 \mathrm{mg} / \mathrm{ml}$ in serum-free medium) was added to the upper side and allowed to polymerize at $37^{\circ} \mathrm{C}$ for $30 \mathrm{~min}$. The OC cells $\left(2 \times 10^{4}\right)$ in $100 \mu \mathrm{l}$ of serum-free medium were added to the upper chamber, and $4 \times 10^{4}$ fibroblasts in $1 \mathrm{ml}$ of culture medium with $0.2 \%$ FBS were added to the lower chamber. Following $24 \mathrm{~h}$ of incubation at $37^{\circ} \mathrm{C}$, the invading cells that had migrated to the opposite side of the chamber membrane were stained with 4',6-diamidino-2-phenylindole and counted in a low-power field (40x magnification). Three fields were counted and the mean was calculated. Images were captured using an Olympus microscope (BX50; Tokyo). Each experiment was performed in triplicate.

Oligonucleotide microarray and analysis. According to the Transwell invasion assay, CAFs from 10 tumors and control fibroblasts isolated from two normal myometrial tissue samples were selected for cDNA microarray analysis. The microarray was performed using the Agilent SurePrint G3 Human High-definition 60K array (Agilent Technologies, Inc.). RNA extraction (RNeasy mini kit, Qiagen $\mathrm{GmbH}$ ) and microarray assays were performed according to standard protocols of the manufacturer. The array results were analyzed using Agilent GeneSpring 7.3 software. The signal was normalized to the 75 th percentile per chip and to the median per gene. The genes were filtered by at least 2 -fold changes compared with the median of all cases. Unsupervised clustering was then analyzed based on these filtered genes. Significance analysis of microarrays was used to identify differentially expressed genes (DEGs) between clustered groups.

RNA interference (RNAi). To knock down the genes of interest, short hairpin (sh)RNA pLKO.1 vectors (National RNAi core facility, Academia Sinica, Taipei, Taiwan) were transfected into 293T cells (ATCC) using a lentiviral system, with the envelope plasmid, pMD.G, and the packaging plasmid, pCMVDR8.91. The shRNA target sequences were as follows: SRPX-1, 5'-CCT ACTGATCTGCCAGTCAAA-3'; SRPX-2, 5'-CCCAGAGAA TGGTTACATGAA-3'; HMCN1-1, 5'-CGCCTGACATTA CATGGCATA-3' and HMCN1-2, 5'-GCCCTCAACATTCCT CATTAT-3'. An shRNA vector against LacZ was used as a 
scrambled control. The transduced cells were selected with puromycin. The RNAi reagents and shRNAs were obtained from the RNAi Core Facility of Academia Sinica.

In vitro overexpression of SRPX. To overexpress SRPX in WS1 cells, the SRPX (GenBank: NM_006307) gene was used in a recombinant lentivirus-expressing vector construction: pLAS2w.Pbsd (Invitrogen; Thermo Fisher Scientific, Inc.). WS1 fibroblasts at a density of $10^{5}$ cells/well were seeded into 24-well plates. Following $24 \mathrm{~h}$ of incubation, $2 \mathrm{ml}$ of fresh media containing $8 \mu \mathrm{g} / \mathrm{ml}$ of polybrene (Sigma-Aldrich; Merck KGaA) and the recombinant lentivirus were added. Obvious green fluorescent protein expression was observed $48 \mathrm{~h}$ after transduction. The WS1 fibroblasts exhibiting SRPX overexpression were then used for the reverse-transcription-quantitative polymerase chain reaction (RT-qPCR), western blot and invasion assays.

$R T-q P C R$ analysis. Total RNA was extracted using TRIzol reagent (Invitrogen; Thermo Fisher Scientific, Inc.) followed by the RNeasy Mini kit (Qiagen GmbHy). The selected DEGs between groups were verified by RT-PCR using an Applied Biosystems (Thermo Fisher Scientific, Inc.) instrument. The probes were designed using Primer 3 software (http://bioinfo.ut.ee/primer3-0.4.0/primer3/). RT-qPCR analysis was performed according to the manufacturer's standard protocols. Total isolated RNA $(1 \mu \mathrm{g})$ was subjected to reverse transcription using PrimeScriptTM RT reagent kit (cat. no. RR037A, Takara Bio). qPCR was performed in triplicate per cDNA sample on an ABI Prism 7900 HT Sequence Detection System (Applied Biosystems; Thermo Fisher Scientific, Inc.) using the FastStart Universal SYBR-Green Master (Roche) and the following running profile: $10 \mathrm{~min}$ enzyme activation at $95^{\circ} \mathrm{C}$, followed by 40 cycles of melting for $10 \mathrm{sec}$ at $95^{\circ} \mathrm{C}$, annealing for $30 \mathrm{sec}$ at $60^{\circ} \mathrm{C}$ and extension for $30 \mathrm{sec}$ at $72^{\circ} \mathrm{C}$. The PCR conditions were optimized to obtain similar efficiencies and calculated from slope for target and reference genes. The primer sequences were as follows: Fibronectin 1 (FN1) forward, 5'-TGGCCAGTCCTACAA CCAGT-3' and reverse, CGGGAATCTTCTCTGTCAGC), SRPX, forward, 5'-CGACTCTCATTGGCAGGATAGGAG-3' and reverse, 5'-ATTCGCAGCAACAGCCTGAG-3', secreted frizzled-related protein 2 (SFRP2), forward, 5'-TGTGGGTCA CAAACCCTGTTGAG-3' and reverse, 5'-AGTCTCAGTCTG GAGCTGATGAAG-3' and hemicentin-1, forward, 5'-AAG GTGGATCATCAGCCGCTTC-3' and reverse, 5'-ACAGTG ACTTTGGGTGGTTCTTGC-3'. Each sample was tested in triplicate, and the cycle difference was calculated using the $2^{-\Delta \Delta C q}$ method (23).

Western blot analysis. The parental WS1 fibroblasts, and the SRPX and HMCN1 shRNA-knockdown WS1 cells $\left(10^{5}\right.$ cells/well) were seeded and collected for western blotting. The cell lysates were prepared as previously described (24). Protein $(30 \mu \mathrm{g})$ from each well was dissolved in loading sample buffer under reducing conditions, separated by $12 \%$ SDS-PAGE, and transferred to $0.2-\mu \mathrm{m}$ nitrocellulose membranes for immunolabelling with the following antibodies: Primary antibodies against SRPX (1:4,000, cat. co. 5473, Cell Signaling Technology, Inc.), RhoA (1:1,000, cat. co. 2117, Cell
Signaling Technology, Inc.), ROCK (1:1,000, cat. co. 4035, Cell Signaling Technology, Inc.), cell division cycle 42 (cdc42) (1:1,000, cat. co. 2466, Cell Signaling Technology, Inc.), MLC (1:500, cat. co. 21157, Cell Signaling Technology, Inc.) and $\beta$-actin $(1: 10,000$, cat. co. MA5-15739, Thermo Fisher Scientific, Inc.) were purchased from Novus Biologicals and incubated at $4^{\circ} \mathrm{C}$ overnight with $\beta$-actin as a loading control. Appropriate secondary antibodies were used to detect proteins as previously described (24). An ImageQuant LAS 4000 analyzer (GE Healthcare Life Sciences) was used to detect protein expression levels.

Statistical analysis. Student's t-test (two-sided) was used to compare Transwell invasion activities between different tumor types. The expression levels of the genes of interest among CAFs with low or high invasion-promoting activities were compared using one-way analysis of variance with Tukey's multiple-comparisons post hoc test. $\mathrm{P}<0.05$ was considered to indicate a statistically significant difference.

\section{Results}

CAFs with high invasion-promoting activities are predominantly from HGSC and CCC samples. In a pilot study, the Transwell invasion activities of several cancer cell lines, including SKOV3, TOV21G, P0 and P4 [P0, endometrioid (EM) carcinoma, and $\mathrm{P} 4$, aggressive cells derived from P0, were provided by Dr PL Torng]. Among these cell lines, the TOV21G line had intermediate Transwell invasion activity and demonstrated the highest discriminatory power in the Transwell invasion assay. Subsequently, the Transwell invasion-inducing activity was compared with a lower chamber that contained culture medium collected following $48 \mathrm{~h}$ of culturing CAFs and that with culture medium containing CAFs. The number of migrated TOV21G cells was higher when the lower chamber contained CAFs rather than medium collected from CAFs after $48 \mathrm{~h}$ of culture (data not shown). Although CAFs can secrete chemotactic factors that induce cancer cell migration, this result suggested that direct crosstalk between cancer cells and CAFs simulating the true TME is important in inducing tumor cell migration. Therefore, the following experiments were performed using the TOV21G cancer cell line and a lower chamber containing CAFs isolated from ovarian tumor tissues.

In total, CAFs from 49 human ovarian tumors following successful Transwell invasion assay were collected, comprising 25 HGSC, 12 CCC, 4 EM carcinoma and 8 mucinous $(\mathrm{Mu})$ tumor (including 5 carcinoma and 3 borderline) samples. In a pilot study, the ratio of cell migration with a lower chamber containing $0.2 \%$ FBS to that containing $10 \%$ FBS was $1: 3$; therefore, the migration number was determined with a lower chamber containing $0.2 \%$ FBS as the internal control in each experiment. According to this definition, the invasion-promoting activities of CAFs were separated into high, intermediate and low according to the number of migrated cancer cells, designated as $>1$-fold, $0.5-1$-fold and $<0.5$-fold, respectively, compared with the internal controls. As shown in Table I, the proportions of CAFs from the HGSC and CCC samples with high invasion-promoting activities were relatively higher compared with those of CAFs from other types 
Table I. Relative Transwell invasion-promoting activity of cancer-associated fibroblasts from different types of ovarian tumor.

\begin{tabular}{lrrrrr}
\hline Activity & HG & CCC & EM & Mu & Total \\
\hline High & 20 & 7 & 1 & 2 & 30 \\
Intermediate & 3 & 4 & 1 & 4 & 12 \\
Low & 2 & 1 & 2 & 2 & 7 \\
Total & 25 & 12 & 4 & 8 & 49 \\
\hline
\end{tabular}

HG, high-grade serous carcinoma; CCC, clear cell carcinoma; $\mathrm{EM}$, endometrioid carcinoma; $\mathrm{Mu}$, mucinous tumor.

of tumors which exhibited either low, intermediate and/or high invasion activities $(\mathrm{P}=0.0085$, Table I). Although $\mathrm{CAFs}$ with low and intermediate tumor invasion-promoting activities were found in HGSC and CCC samples, there were fewer than the number with high invasion-promoting activities. These results are consistent with clinical observations that aggressive ovarian tumors are usually of HGSC and CCC types, whereas $\mathrm{EM}$ and $\mathrm{Mu}$ types tend to be indolent tumors.

CAFs from different OC types have different expression profiles. To identify potential factors in CAFs that contribute to their invasion-promoting activities, the present study compared the expression profiles of CAFs with high and low invasion-promoting activities by microarray analysis. A total of 10 different CAFs were selected, including two HGSCs with high invasion-promoting activity $\left(\mathrm{HG}^{+}\right)$, two $\mathrm{HGSCs}$ with low invasion-promoting activity $\left(\mathrm{HG}^{-}\right)$, two $\mathrm{CCCs}$ with high invasion-promoting activity $\left(\mathrm{CCC}^{+}\right)$, two $\mathrm{CCCs}$ with low invasion-promoting activity ( $\left.\mathrm{CCC}^{-}\right)$, two EMs with low invasion-promoting activity $\left(\mathrm{EM}^{-}\right)$and two normal fibroblasts (NOFs) (Fig. S1B). Principal component analysis (PCA) of the raw array data (Fig. S1A) showed that the two NOFs had similar expression patterns, whereas the $10 \mathrm{CAFs}$ presented with various expression profiles, possibly due to inherent tumor heterogeneity and the variations of raw flag signals at individual probes. However, there was a vague clustering of CAFs from the HGSC and EM samples, whereas the CAFs from CCC samples tended to have a wider range of expression profiles, suggesting that different histological types may contribute to different expression profiles. Following normalization of the signals and filtering of the genes, 369 genes with at least 2-fold changes were selected for clustering analysis. On comparing the DEGs between CAFs obtained from $\mathrm{HG}^{+}$ and $\mathrm{HG}^{-}$cases, 92 genes were upregulated and 52 genes were downregulated by at least 2-fold. In CAFs obtained from $\mathrm{CCC}^{+}$ and $\mathrm{CCC}^{-}$cases, 69 genes were upregulated and 37 genes were downregulated by at least 2 -fold. Selective significantly upregulated and downregulated genes are listed for HGSC samples in Tables II and SI, respectively. Certain significantly DEGs found in CAFs from both HGSC and CCC samples were membrane-form or secreted proteins and are reported to be related to the aggressiveness and/or invasiveness of various types of cancer, including IL-6 (13), FN1, pleiotrophin, fibroblast growth factor 7, SFRP2 and neuropilin 2 .
Table II. Upregulated genes in cancer-associated fibroblasts with high Transwell invasion-promoting activity from high-grade serous carcinomas.

\begin{tabular}{ll} 
Gene & Fold-change \\
\hline Loc100288985 & 21.834822 \\
GRINL1A complex locus & 19.478653 \\
Breast carcinoma-amplified sequence 1 & 19.120928 \\
Family with sequence similarity 150, & 12.909669 \\
number B & \\
RFPL1 antisense RNA (non-protein coding) & 8.519261 \\
Hemicentin 1 & 7.490842 \\
Anoctamin 2 & 5.058346 \\
Sushi repeat-containing protein, X-linked & 4.719756 \\
TAF9B RNA polymerase II & 4.568907 \\
Ectonucleotide pyrophosphatase/ & 4.362322 \\
phosphodiesterase 6 & \\
Fibronectin 1 & 3.948831 \\
Transmembrane protein 155 & 3.911077 \\
Ras-related associated with diabetes & 3.875658 \\
Monocarboxylic acid transporter 12 & 3.852940 \\
Actin-binding LIM protein family, member 2 & 3.851354 \\
\hline
\end{tabular}

Expression of representative genes identified from a microarray in CAFs with high and low invasion-promoting activities obtained from HGSC and CCC samples and confirmed by RT-qPCR analysis. As HGSC is the most common type of OC with a poor prognosis, four DEGs were selected, including three upregulated genes (SRPX, HMCN1 and FN1) and one downregulated gene (SFRP2), detected in CAFs from HGSC samples for further verification. Therefore, the expression levels of these four genes were compared among CAFs with different invasion-promoting activities. In total, 47 CAFs were included in the RT-qPCR analysis, in which 24, 14 and nine samples had high, intermediate and low invasion-promoting activities, respectively. When CAFs from all histological types were included, a significant difference in expression was observed for SRPX between CAFs with high and low invasion-promoting activities $(\mathrm{P}=0.01$, data not shown), whereas no significant differences were observed for other genes. However, when comparing CAFs from only HGSC and CCC samples, significant differences in expression levels were observed for SRPX and HMCN1 between CAFs with high low invasion-promoting activities (Fig. 1A and $\mathrm{B}, \mathrm{P}=0.02$ and 0.009 , respectively), whereas no significant difference was observed in the expression of FN1 or SFRP2 (Fig. 1C and D).

Inhibition of OC cell migration and invasion through downregulating SRPX and/or HMCN1 in WS1 fibroblast cells via the RhoA signaling pathway. In order to further confirm whether the SRPX and HMCN1 genes in CAFs affect the invasiveness of OC cells, SRPX and HMCN1 were knocked down using an shRNA approach in WS1 fibroblasts. The results showed that the mRNA expression levels of SRPX were successfully reduced by $>90 \%(\mathrm{P}<0.001)$ in WS1 cells 

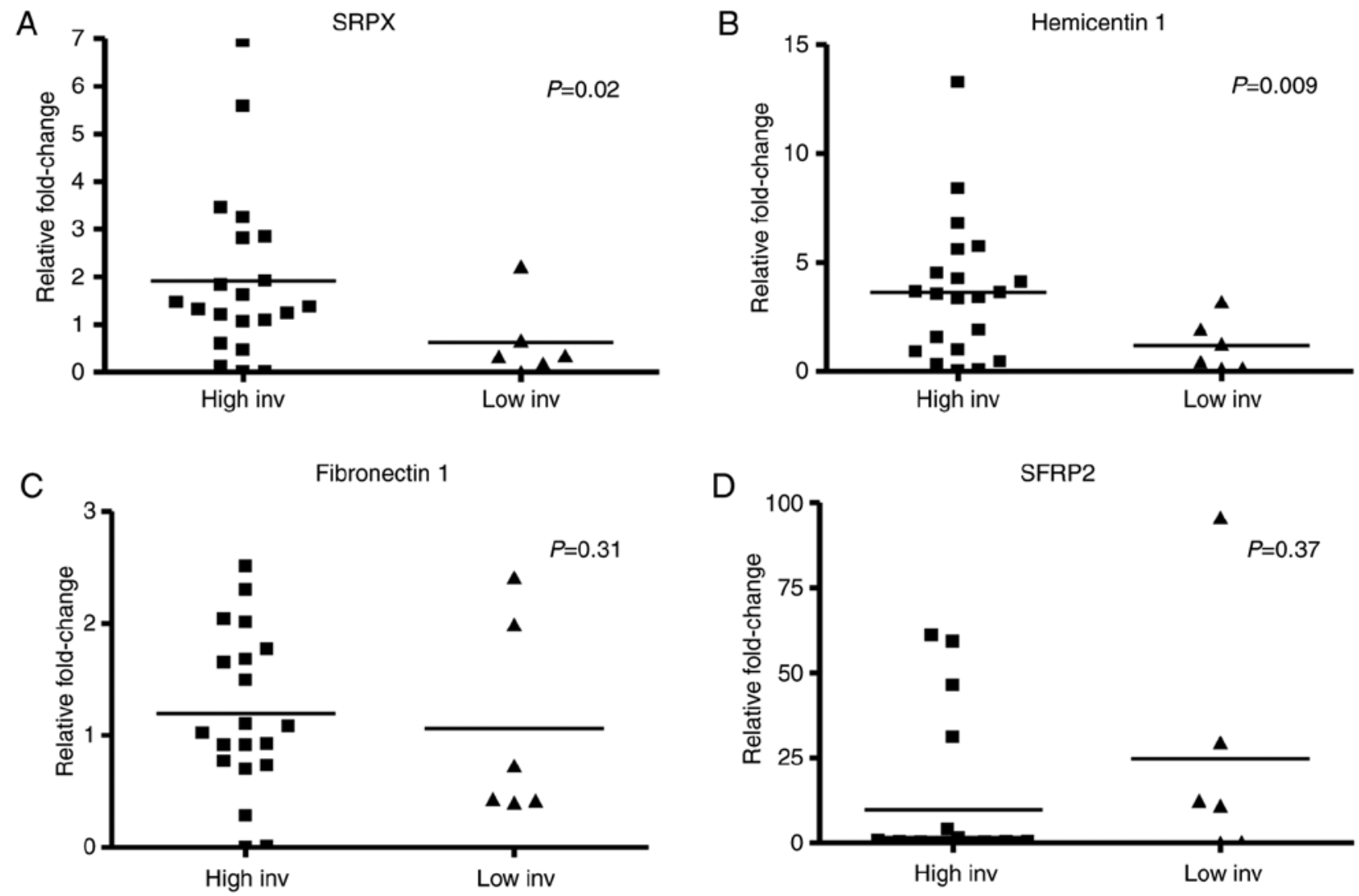

Figure 1. Expression of representative genes using RT-qPCR analysis. (A) $S R P X$, (B) $H M C N 1$, (C) fibronectin 1 and (D) $S F R P 2$ genes identified from a microarray assay in CAFs with high and low invasion-promoting activities from high-grade serous carcinoma and clear cell carcinoma samples were compared. mRNA levels of SRPX and HMCN1 were significantly higher ( $\mathrm{P}=0.02$ and $\mathrm{P}=0.009$, respectively) in CAFs with high invasion-promoting activities as confirmed by an RT-qPCR assay. CAFs, cancer-associated fibroblasts; SRPX, sushi repeat-containing protein, X; HMCN1, hemicentin 1; SFRP2, secreted frizzled-related protein 2; RT-qPCR, reverse-transcription-quantitative polymerase chain reaction; inv, invasion.

transfected with either the shRNA-SRPX-1 (clone SRPX-1) or shRNA-SRPX-2 (clone SRPX-2). The mRNA expression levels of $H M C N 1$ were also reduced with similar efficacies ( $\mathrm{P}<0.001)$ using either shRNA-HMCN1-1 (clone HMCN1-1) or shRNA-HMCN1-2 (clone HMCN1-2), compared with levels in the untransfected WS1 cells (Fig. 2A and C). Following SRPX and HMCN1 silencing, the abilities of WS1 cells to promote TOV21G migration and invasion were elucidated using an in vitro wound-healing assay and invasion assay. Compared with the parental WS1 cells, the downregulation of SRPX using either the SRPX-1 or SRPX-2 clone led to a significant decrease $(\mathrm{P}<0.001$; Figs. $2 \mathrm{~B}$ and $\mathrm{S} 2)$ in the migration and Transwell invasion of TOV21G cells, which indicated that the invasive ability of the TOV21G cells was closely associated with SRPX-expressing CAFs. Similarly, the knockdown of $H M C N 1$ in WS1 cells using the HMCN1-1 or HMCN1-2 clone significantly reduced the migration and Transwell invasion of TOV21G cells over an incubation period of $24 \mathrm{~h}$ compared with that in the mock-transfected and parental cells $(\mathrm{P}<0.001$; Figs. 2D and S2). Of note, there was no suppression of Transwell invasion when the lower chamber contained medium collected from cultured SRPX- or HMCNI-knockdown WS1 cells (data not shown). This observation is similar to that of our preliminary data and reiterated the significance of the direct interaction between CAFs and tumor cells in the TME.

The potential mechanism of SRPX and HMCNI in stimulating TOV21G cell invasion activities were subsequently investigated in vitro using RT-qPCR and western blot assays. The results showed that the mRNA levels of $S R P X$ were significantly reduced in the $S R P X$ - and $H M C N 1$-knockdown WS1 fibroblasts $(\mathrm{P}<0.001$; Fig. 3A); furthermore, the mRNA levels of $H M C N 1$ were significantly reduced in the $S R P X$ and HMCN1-knockdown WS1 cells compared with those in the mock transfection and parental cells $(\mathrm{P}<0.001$; Fig. 3B). In addition, the knockdown of SRPX or HMCN1 in WS1 cells reduced the levels of ROCK, RhoA, cdc42 and MLC (Fig. 3C), suggesting that the invasiveness of OC cells is regulated by the RhoA/ROCK/MLC and cdc42 signaling pathways.

Overexpression of SRPX in WS1 fibroblasts stimulates OC cell invasion ability in vitro. To further investigate the role of SRPX-fibroblasts involved in OC cell invasion, the effects of the overexpression of SRPX in WS1 cells was investigated. The mRNA levels of SRPX and HMCNI $(\mathrm{P}<0.001$; Fig. 4A) and the protein levels of SRPX, ROCK, RhoA and MLC (Fig. 4B) were markedly increased in WS1 fibroblasts transduced with the SRPX recombinant lentivirus. In the Transwell invasion assay, a markedly increased TOV21G invasive ability was observed in WS1 fibroblasts overexpressing SRPX during the 12-h incubation period ( $\mathrm{P}<0.001$; Fig. $4 \mathrm{C})$ compared with that in the mock transduction and parental control groups. These results suggest that SRPX is an important factor in CAFs regulating the invasiveness of $\mathrm{OC}$ cells. 


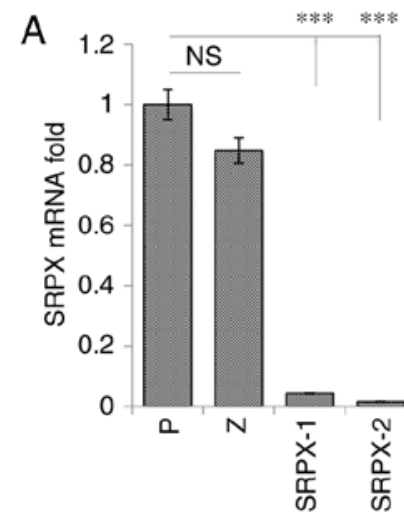

B
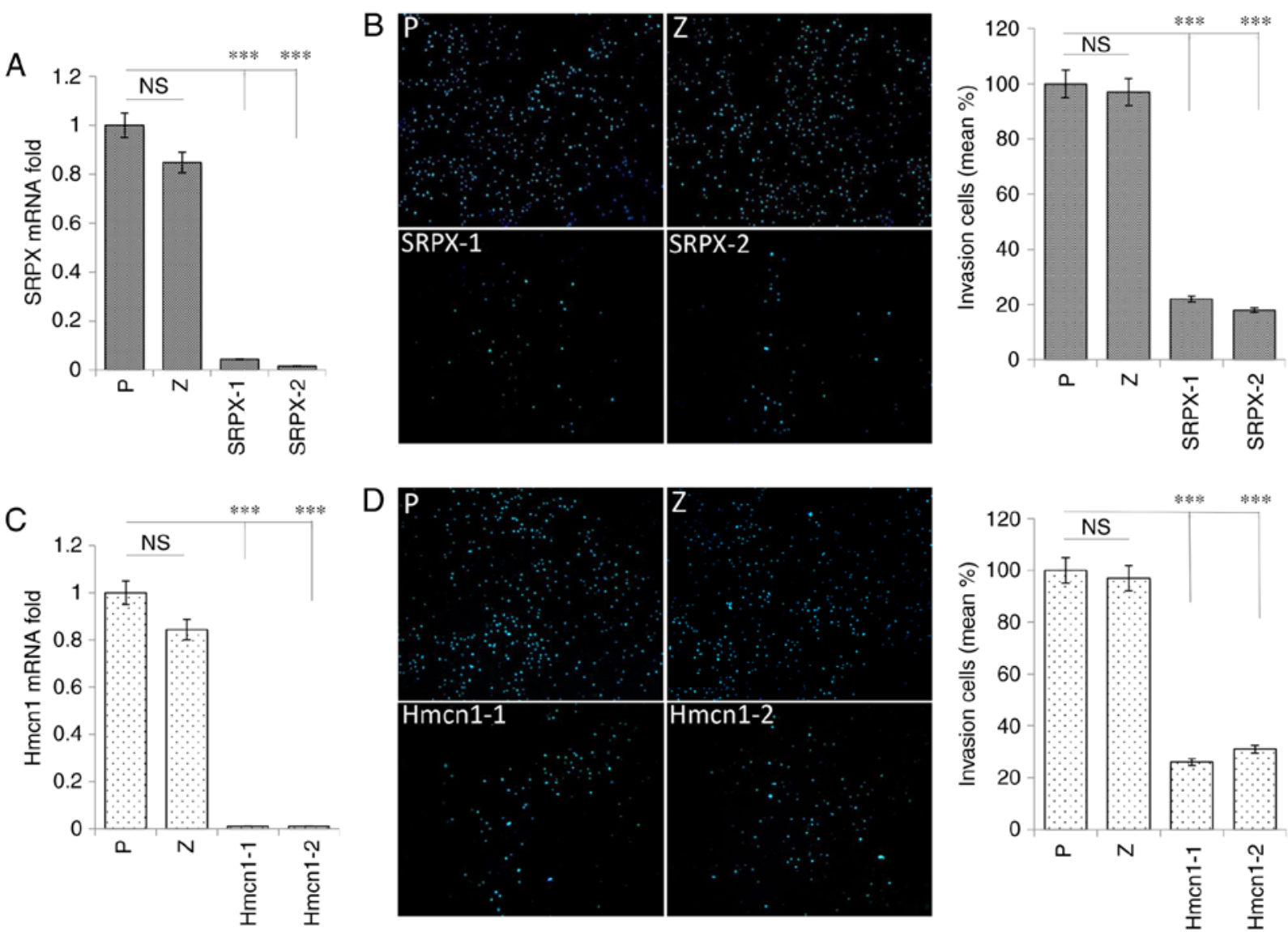

Figure 2. SRPX and HMCN1 shRNA in WS1 fibroblast cells suppress TOV21G ovarian cancer cell invasion in vitro. Compared with mock transduction and normal WS1 cells, SRPX shRNA-knockdown WS1 cells (SRPX-1 and SRPX-2) showed (A) significantly decreased mRNA levels according to the RT-qPCR assay and (B) reduced promotion of invasion in an in vitro Transwell invasion assay. HMCN1 shRNA-knockdown WS1 cells (HMCN1-1 and HMCN1-2) also showed (C) A significantly decreased mRNA levels according to the RT-qPCR assay and (D) significantly reduced promotion of invasion in an in vitro Transwell invasion assay. Data are expressed as the mean \pm standard deviation $\left({ }^{* * *} \mathrm{P}<0.001\right)$. Similar results were obtained from at least three independent experiments. P, parental (normal WS1) cells; Z, mock-transduced; SRPX, sushi repeat-containing protein, X; HMCN1, hemicentin 1; shRNA, short hairpin RNA; RT-qPCR, reverse-transcription-quantitative polymerase chain reaction; ns, not significant.

\section{Discussion}

CAFs are important components of the TME and serve critical roles in cancer progression, metastasis and therapeutic resistance (25). Despite their well-known significance in tumors, the molecular characteristics of CAFs specifically in OC remain to be fully elucidated. The present study has several important implications although with some limitations. First, OCs are heterogeneous with the pathogenesis and prognosis differing among different histological types, therefore, the present study first compared the invasion-promoting activities of CAFs from different histological types of OC. The results revealed for the first time, to the best of our knowledge, that CAFs from HGSC and CCC samples exhibited significantly higher invasion-promoting activities compared with CAFs from other types of ovarian tumors. This result is consistent with clinical observations that aggressive ovarian tumors with high mortality rates are mostly HGSCs and CCCs $(26,27)$. Second, the results revealed that the presence of conditioned medium collected from CAFs, but without CAFs, exerted no effect on tumor behavior, thus emphasizing the importance of close contact between OCs and CAFs in the TME to exert pathological effects. It is important to note that the Transwell system is a $2 \mathrm{D}$ rather than a $3 \mathrm{D}$ system, which is more akin to the real TME. Third, there is a limited number of studies on OC CAFs via a wide scale molecular approach. Two previous studies focused on comparing the expression profiles of microRNAs (28) and long non-coding RNAs (29) between CAFs and normal fibroblasts. The present study was unique in that, in addition to the inclusion of normal fibroblasts, CAFs exhibiting different phenotypes were compared. The PCA clustering result revealed that the well-known tumor heterogeneity is also observed in CAFs. This CAF heterogeneity phenomenon is also present in carcinomas from other sites (30) and has been discussed in several review articles $(31,32)$. By comparing expression profiles of CAFs with high and low invasion-promoting activities, the present study found that SRPX and HMCN1 were significantly overexpressed in CAFs from HGSC and CCC samples with high invasion-promoting activities. In addition, SRPX and HMCN1 silencing in WS1 cells led to reduced tumor cell invasion and a reduction in the expression of RhoA, likely through the RhoA/ROCK/MLC signaling pathway, which is reported to be involved in regulating cell invasion and tumor metastasis (33-35). These results support that $S R P X$ and $H M C N 1$ can serve as potential therapeutic targets in CAFs for treating OC.

SRPX, also known as SRPX1 (36), ETX1 (37) and DRS (38), is a transmembrane protein consisting of 464 amino 
A

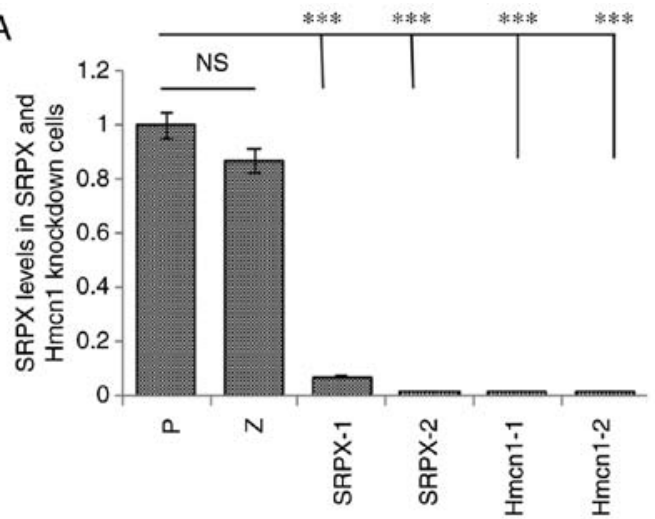

B

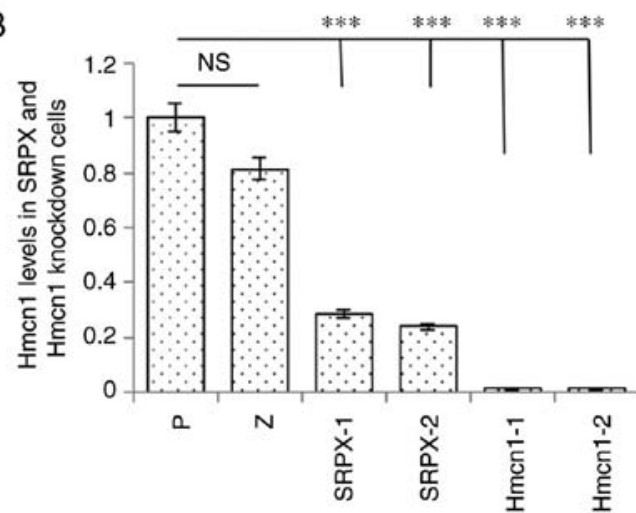

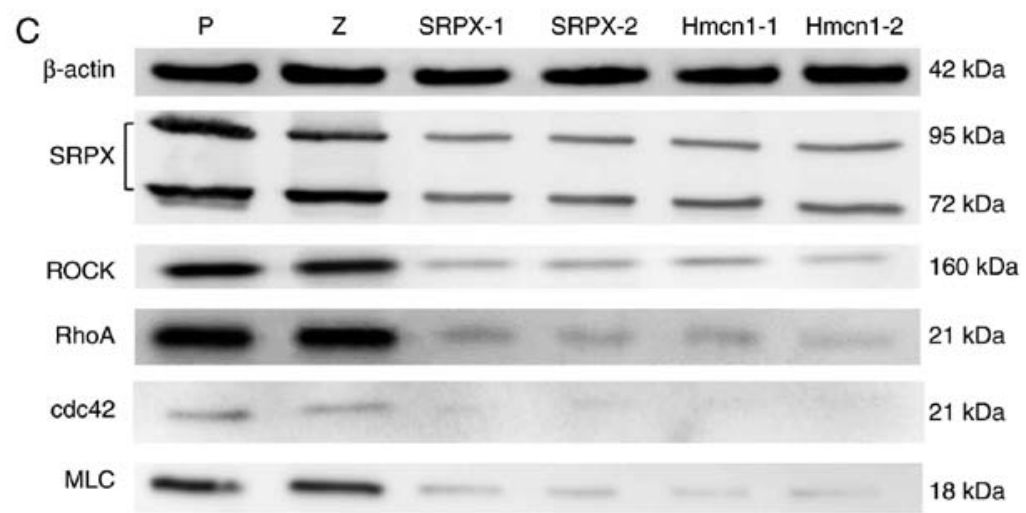

Figure 3. Knockdown of SRPX and HMCN1 in WS1 cells significantly reduced TOV21G cell invasion through the RhoA signaling pathway. Representative multiples of changes in comparisons of reverse-transcription-quantitative polymerase chain reaction results for (A) SRPX and (B) $H M C N 1$ ( $\left.{ }^{* * *} \mathrm{P}<0.001\right)$. (C) Images of western blot analysis of the expression of SRPX, HMCN1, ROCK, RhoA, cde42 and MLC in WS1 parental, mock-transduced, SRPX-downregulated cells (SRPX-1 and SRPX-2) and HMCN1-downregulated cells (HMCN1-1 and HMCN1-2). Similar results were obtained from at least three independent experiments. Western blot analysis of HMCN1 was not performed due to no commercially available antibody. SRPX, sushi repeat-containing protein, X; HMCN1, hemicentin 1; P, parental (normal WS1) cells; Z, mock-transduced; RhoA, Ras homology family member A; ROCK, Rho kinase; cdc42, cell division cycle 42; MLC, myosin II regulatory light chain; ns, not significant.

A

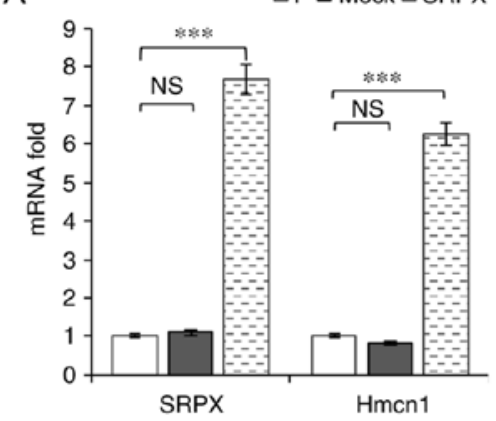

B

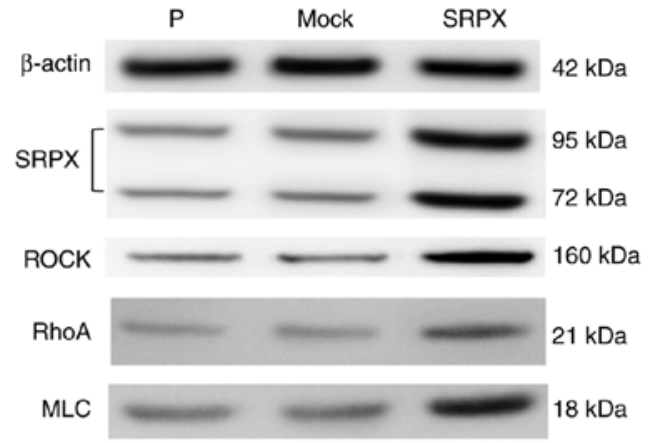

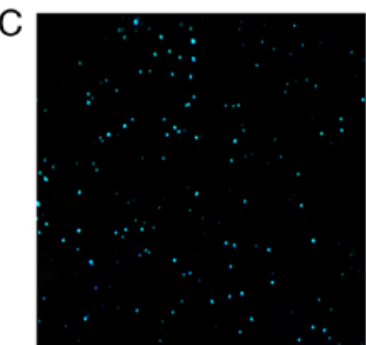

$\mathrm{P}$

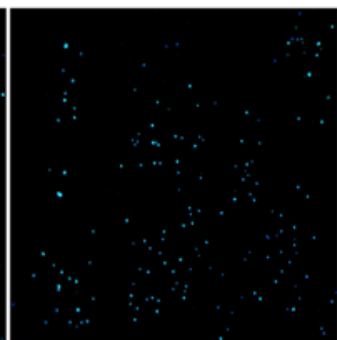

Mock

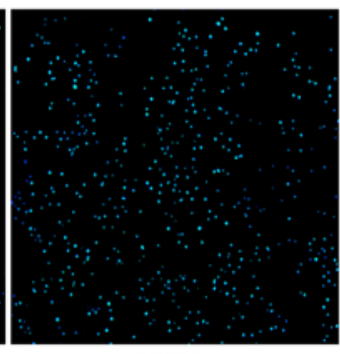

SRPX

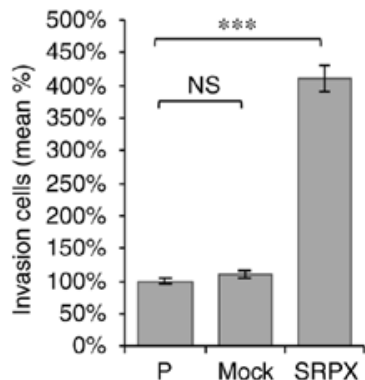

Figure 4. Overexpression of SRPX in WS1 fibroblasts induces TOV21G cell invasion. Representative (A) changes of SRPX and HMCN1 detected by reverse-transcription-quantitative polymerase chain reaction analysis. (B) images of the western blots of the expression of SRPX, ROCK, RhoA and MLC in WS1 parental, mock-transduced and SRPX-overexpressing fibroblasts. (C) Overexpression of SRPX in WS1 fibroblasts increased TOV21G cell invasion abilities $\left({ }^{* * *} \mathrm{P}<0.001\right)$. The experiment was repeated at least three times. SRPX, sushi repeat-containing protein, X; HMCN1, hemicentin 1 ; $\mathrm{P}$, parental (normal WS1) cells; RhoA, Ras homology family member A; ROCK, Rho kinase; cdc42, cell division cycle 42; MLC, myosin II regulatory light chain; ns, not significant. 
acids and three sushi domains. It is reported to be downregulated in a variety of human tumor cells and tissues, and is thus considered to be a tumor-suppressor gene (38). This was confirmed by evidence showing that $\sim 30 \%$ of $\operatorname{Srpx}$-knockout mice generated various tumors, including lymphomas, lung cancer and hepatomas (38). SRPX was initially identified as a causative gene in patients with X-linked retinitis pigmentosa, and a recent study revealed that SRPX is a novel disease-associated molecule in cerebral amyloid angiopathy (36). Although $S R P X$ is known to be a tumor-suppressor gene, the present study is the first, to the best of our knowledge, to reveal its significant upregulation in HGSC and CCC CAFs, which was further evidenced by the stimulation of OC invasion via SRPX knockdown in WS1 cells in vitro.

HMCN1 (also known as fibulin-6) (39) was first found in Caenorhabditis elegans, was named high incidence of males 4 and is one of the genes responsible for increased $\mathrm{X}$ chromosome loss in nematodes (40). Functionally, HMCN1 is associated with a hemidesmosome-type structure and is involved in extracellular adhesion, forming cell-cell and cell-basement membrane adhesions that hold cells together and maintain tissue and organ integrity (41). Therefore, the loss of $H M C N 1$ leads to multinucleate cells, as previously observed in $C$. elegans germ lines which exhibited occasional fusion of neighboring cells, whereas HMCNl deficiency in mice produced incomplete mitotic cytokinesis of mice embryonic cells (41). Studies in humans have shown that deficiencies of HMCN1 were associated with age-related macular degeneration (42), Fraser syndrome (43) and glomerular diseases (44), which were all correlated with the mechanisms of basement membrane anchorage functions of $H M C N 1$. However, few studies have focused on the functions of $H M C N 1$ in tumorigenesis. The present study found that $H M C N 1$ was significantly upregulated in HGSC and CCC CAFs, and silencing HMCNI by shRNA in fibroblasts markedly reduced the invasive ability of TOV21G cells.

CAFs are crucial cellular components of the desmoplastic stroma characteristic of the TME, and various cellular pathways are implicated in regulating CAF functions. Among these, several secreted molecular regulators of CAFs have been shown to be involved in promoting tumor growth, such as platelet-derived growth factors, epidermal growth factors, fibroblast growth factors and sonic hedgehog $(45,46)$. CAFs also have the ability to invade through the matrix and are widely reported to promote the invasiveness of cancer cells (47), by mediating chemicals such as galectin-1, which upregulates matrix metalloproteinase- 9 and thereby promotes breast cancer cell metastasis (48), and E-cadherin/N-cadherin activation, which enables fibroblasts to drive cancer cell invasion (49). On investigating the underlying mechanisms, the present found that the silencing of SRPX and HMCN1 in fibroblasts was associated with inhibition of the RhoA/ROCK/MLC pathway. This is supported by evidence that the activation of RhoA/ROCK signaling via SMAD-specific E3 ubiquitin ligase can promote OC cell migration and invasion (34). Studies have also shown that the RhoA/ROCK pathway is involved in tight junctions in different cell types, which facilitate their permeability and attachment functions $(33,35)$.

In conclusion, the present study demonstrated that the CAF-associated proteins, SRPX and HMCN1, were significantly upregulated and correlated with promoting the migratory activities of OC cells. The results also confirmed that the silencing of $S R P X$ and $H M C N 1$ markedly suppressed OC invasion via inhibiting the RhoA/ROCK/MLC signaling pathway. As CAFs contribute towards such a critical aspect essential for tumor progression, particularly tumor invasiveness, strategies targeting CAFs to treat $\mathrm{OC}$ may be effective. In addition to conventional treatments targeting tumor cells, these findings highlight the critical role of CAF-associated SRPX and $\mathrm{HMCN} 1$ in promoting $\mathrm{OC}$ invasion and indicate that both molecules represent potential targets for OC treatment.

\section{Acknowledgements}

The authors would like to thank the National RNAi Core Facility at Academia Sinica in Taiwan for providing shRNA reagents and related services.

\section{Funding}

This study was supported in part by grants (grant nos. NSC98-2320-B-002-041-MY3 and MOST105-2628-B038-009-MY3) from the Ministry of Science and Technology, Taiwan.

\section{Availability of data and materials}

All data generated and/or analyzed during the present study are included in this published article and its supplementary information files.

\section{Authors' contributions}

TLM designed the research project, analyzed data, and wrote the manuscript. CLL designed the project and wrote the manuscript. HWP initiated the project and helped design the Transwell assay. PLT provided the in-house cell line and helped design the project. MHF performed the experiments.

\section{Ethics approval and consent to participate}

The use of human materials was approved by the Ethics Committee and Review Board at National Taiwan University Hospital (IRB approval no. 200812141R). Individual written informed consent was obtained from all patients.

\section{Patient consent for publication}

Not applicable.

\section{Competing interests}

The authors declare that they have no competing interests.

\section{References}

1. Peres LC, Cushing-Haugen KL, Anglesio M, Wicklund K, Bentley R, Berchuck A, Kelemen LE, Nazeran TM, Gilks CB, Harris HR, et al: Histotype classification of ovarian carcinoma: A comparison of approaches. Gynecol Oncol 151: 53-60, 2018 
2. Kurman RJ, Carcangiu ML, Herrington CS and Young RH: WHO classification of tumours of female reproductive organs 4th Edition, pp12, 2014.

3. Bowtell DD, Böhm S, Ahmed AA, Aspuria PJ, Bast RC Jr, Beral V, Berek JS, Birrer MJ, Blagden S, Bookman MA, et al: Rethinking ovarian cancer II: Reducing mortality from high-grade serous ovarian cancer. Nat Rev Cancer 5: 668-679, 2015.

4. Wang W, Kryczek I, Dostál L, Lin H, Tan L, Zhao L, Lu F, Wei S, Maj T, Peng D, et al: Effector T cells abrogate Stroma-mediated chemoresistance in ovarian cancer. Cell 165 : 1092-1105, 2016.

5. Binju M, Padilla MA, Singomat T, Kaur P, Suryo Rahmanto Y, Cohen PA and Yu Y: Mechanisms underlying acquired platinum resistance in high grade serous ovarian cancer-a mini review. Biochim Biophys Acta Gen Subj 1863: 371-378, 2019.

6. Wu W, Wang Q, Yin F, Yang Z, Zhang W, Gabra H and Li L: Identification of proteomic and metabolic signatures associated with chemoresistance of human epithelial ovarian cancer. Int J Oncol 49: 1651-1665, 2016

7. Augsten M: Cancer-associated fibroblasts as another polarized cell type of the tumor microenvironment. Front Oncol 4: 62, 2014

8. Tommelein J, Verset L, Boterberg T, Demetter P, Bracke M and De Wever O: Cancer-associated fibroblasts connect metastasis-promoting communication in colorectal cancer. Front Oncol 5: 63, 2015.

9. Schauer IG, Sood AK, Mok S and Liu J: Cancer-associated fibroblasts and their putative role in potentiating the initiation and development of epithelial ovarian cancer. Neoplasia 13: 393-405, 2011.

10. Paulsson $\mathrm{J}$ and Micke P: Prognostic relevance of cancer-associated fibroblasts in human cancer. Semin Cancer Biol 25: 61-68, 2014.

11. Jahangiri B, Khalaj-Kondori M, Asadollahi E and Sadeghizadeh M: Cancer-associated fibroblasts enhance cell proliferation and metastasis of colorectal cancer SW480 cells by provoking long noncoding RNA UCA1. J Cell Commun Signal 13: 53-64, 2019.

12. Emon B, Bauer J, Jain Y, Jung B and Saif T: Biophysics of tumor microenvironment and cancer metastasis-A mini review. Comput Struct Biotechnol J 16: 279-287, 2018.

13. Qin X, Yan M, Wang X, Xu Q, Wang X, Zhu X, Shi J, Li Z, Zhang J and Chen W: Cancer-associated fibroblast-derived IL-6 promotes head and neck cancer progression via the osteopontin-NF-kappa B signaling pathway. Theranostics 8: 921-940, 2018.

14. Miki Y, Yashiro M,Okuno T, Kitayama K, Masuda G, Hirakawa K and Ohira M: CD9-positive exosomes from cancer-associated fibroblasts stimulate the migration ability of scirrhous-type gastric cancer cells. Br J Cancer 118: 867-877, 2018.

15. Li W, Zhang X, Wang J, Li M, Cao C, Tan J, Ma D and Gao Q: TGF $\beta 1$ in fibroblasts-derived exosomes promotes epithelial-mesenchymal transition of ovarian cancer cells Oncotarget 8: 96035-96047, 2017.

16. Han Y, Zhang Y, Jia T and Sun Y: Molecular mechanism underlying the tumor-promoting functions of carcinoma-associated fibroblasts. Tumour Biol 36: 1385-1394, 2015

17. Österreicher CH, Penz-Österreicher M, Grivennikov SI Guma M, Koltsova EK, Datz C, Sasik R, Hardiman G, Karin M and Brenner DA: Fibroblast-specific protein 1 identifies an inflammatory subpopulation of macrophages in the liver. Proc Natl Acad Sci USA 108: 308-313, 2011.

18. Higashino N, Koma YI, Hosono M, Takase N, Okamoto M Kodaira H, Nishio M, Shigeoka M, Kakeji Y and Yokozaki H: Fibroblast activation protein-positive fibroblasts promote tumor progression through secretion of CCL2 and interleukin-6 in esophageal squamous cell carcinoma. Lab Invest 99: 777-792, 2019.

19. Kratochwil C, Flechsig P, Lindner T, Abderrahim L, Altmann A, Mier W, Adeberg S, Rathke H, Roehrich M, Winter H, et al: FAPI-PET/CT: Mean intensity of tracer-uptake (SUV) in 28 different kinds of cancer. J Nucl Med: April 6, 2019.

20. Leung CS, Yeung TL, Yip KP, Wong KK, Ho SY, Mangala LS, Sood AK, Lopez-Berestein G, Sheng J, Wong ST, et al: Cancer-associated fibroblasts regulate endothelial adhesion protein LPP to promote ovarian cancer chemoresistance. J Clin Invest 128: 589-606, 2018.

21. Zhang Y, Tang H, Cai J, Zhang T, Guo J, Feng D and Wang Z: Ovarian cancer-associated fibroblasts contribute to epithelial ovarian carcinoma metastasis by promoting angiogenesis, lymphangiogenesis and tumor cell invasion. Cancer Lett 303: $47-55,2011$
22. Hosein AN, Wu M, Arcand SL, Lavallée S, Hébert J, Tonin PN and Basik M: Breast carcinoma-associated fibroblasts rarely contain p53 mutations or chromosomal aberrations. Cancer Res 70: 5770-5777, 2010.

23. Livak KJ and Schmittgen TD: Analysis of relative gene expression data using real-time quantitative PCR and the 2(-Delta Delta C(T)) method. Methods 25: 402-408, 2001

24. Mao TL, Fan KF and Liu CL: Targeting the CXCR4/CXCL12 axis in treating epithelial ovarian cancer. Gene Ther 24: 621-629, 2017.

25. Dasari S, Fang Y and Mitra AK: Cancer Associated Fibroblasts Naughty neighbors that drive ovarian cancer progression. Cancers (Basel) 10: E406, 2018.

26. Hogen L, Vicus D, Ferguson SE, Gien LT, Nofech-Mozes S, Lennox GK and Bernardini MQ: Patterns of recurrence and impact on survival in patients with clear cell ovarian carcinoma. Int J Gynecol Cancer 29: 1164-1169, 2019.

27. Fortner RT, Poole EM, Wentzensen NA, Trabert B, White E, Arslan AA, Patel AV, Setiawan VW, Visvanathan K, Weiderpass E, et al: Ovarian cancer risk factors by tumor aggressiveness: An analysis from the ovarian cancer cohort consortium. Int J Cancer 145: 58-69, 2019.

28. Mitra AK, Zillhardt M, Hua Y, Tiwari P, Murmann AE, Peter ME and Lengyel E: MicroRNAs reprogram normal fibroblasts into cancer-associated fibroblasts in ovarian cancer. Cancer Discov 2 1100-1108, 2012

29. Vafaee F, Colvin EK, Mok SC, Howell VM and Samimi G: Functional prediction of long non-coding RNAs in ovarian cancer-associated fibroblasts indicate a potential role in metastasis. Sci Rep 7: 10374, 2017.

30. Manousopoulou A, Hayden A, Mellone M, Garay-Baquero DJ, White CH, Noble F, Lopez M, Thomas GJ, Underwood TJ and Garbis SD: Quantitative proteomic profiling of primary cancer-associated fibroblasts in oesophageal adenocarcinoma. Br J Cancer 118: 1200-1207, 2018.

31. Ishii G, Ochiai A and Neri S: Phenotypic and functional heterogeneity of cancer-associated fibroblast within the tumor microenvironment. Adv Drug Deliv Rev 99: 186-196, 2016.

32. Ohlund D, Elyada E and Tuveson D: Fibroblast heterogeneity in the cancer wound. J Exp Med 211: 1503-1523, 2014.

33. Soliman M, Cho EH, Park JG, Kim JY, Alfajaro MM, Baek YB, Kim DS, Kang MI, Park SI and Cho KO: Rotavirus-induced early activation of the RhoA/ROCK/MLC signaling pathway mediates the disruption of tight junctions in polarized MDCK cells. Sci Rep 8: 13931, 2018.

34. Wang W, Du H, Liu H, Hu F and Liu G: SMAD specific E3 ubiquitin protein ligase 1 promotes ovarian cancer cell migration and invasion via the activation of the RhoA/ROCK signaling pathway. Oncol Rep 41: 668-676, 2019.

35. Feng S, Zou L, Wang H, He R, Liu K and Zhu H: RhoA/ROCK-2 pathway inhibition and tight junction protein upregulation by catalpol suppresses lipopolysaccaride-induced disruption of blood-brain barrier permeability. Molecules 23: pii: E2371, 2018.

36. Inoue $\mathrm{Y}$, Ueda M, Tasaki M, Takeshima A, Nagatoshi A, Masuda T, Misumi Y, Kosaka T, Nomura T, Mizukami M, et al: Sushi repeat-containing protein 1: A novel disease-associated molecule in cerebral amyloid angiopathy. Acta Neuropathol 134: 605-617, 2017.

37. Iragavarapu S, Algeciras ME, Lee RK and Bhattacharya SK: ETX1 is over-expressed in the glaucomatous trabecular meshwork. Mol Vis 15: 2061-2067, 2009.

38. Tambe Y, Hasebe M, Kim CJ, Yamamoto A and Inoue H: The drs tumor suppressor regulates glucose metabolism via lactate dehydrogenase-B. Mol Carcinog 55: 52-63, 2016.

39. Chowdhury A, Hasselbach L, Echtermeyer F, Jyotsana N, Theilmeier $\mathrm{G}$ and Herzog C: Fibulin-6 regulates pro-fibrotic TGF- $\beta$ responses in neonatal mouse ventricular cardiac fibroblasts. Sci Rep 7: 42725, 2017.

40. Lan H, Wang X, Jiang L, Wu J, Wan X, Zeng L, Zhang D, Lin Y, Hou C, Wu S and Tse YC: An extracellular matrix protein promotes anillin-dependent processes in the Caenorhabditis elegans germline. Life Sci Alliance 2: pii: e201800152, 2019.

41. Xu X and Vogel BE: A secreted protein promotes cleavage furrow maturation during cytokinesis. Curr Biol 21: 114-119, 2011.

42. Pras E, Kristal D, Shoshany N, Volodarsky D, Vulih I, Celniker G, Isakov O, Shomron N and Pras E: Rare genetic variants in Tunisian Jewish patients suffering from age-related macular degeneration. J Med Genet 52: 484-492, 2015. 
43. Carney TJ, Feitosa NM, Sonntag C, Slanchev K, Kluger J, Kiyozumi D, Gebauer JM, Coffin Talbot J, Kimmel CB, Sekiguchi K, et al: Genetic analysis of fin development in zebrafish identifies furin and hemicentin1 as potential novel fraser syndrome disease genes. PLoS Genet 6: e1000907, 2010.

44. Toffoli B, Zennaro C, Winkler C, Giordano Attianese GMP, Bernardi S, Carraro M, Gilardi F and Desvergne B: Hemicentin 1 influences podocyte dynamic changes in glomerular diseases. Am J Physiol Renal Physiol 314: F1154-F1165, 2018.

45. Chen JS, Liang LL, Xu HX, Chen F, Shen SL, Chen W, Chen LZ, Su Q, Zhang LJ, Bi J, et al: miR-338-3p inhibits epithelial-mesenchymal transition and metastasis in hepatocellular carcinoma cells. Oncotarget 8: 71418-71429, 2016.

46. Xing F, Saidou J and Watabe K: Cancer associated fibroblasts (CAFs) in tumor microenvironment. Front Biosci (Landmark Ed) 15: 166-179, 2010
47. Hanahan D and Coussens LM: Accessories to the crime: Functions of cells recruited to the tumor microenvironment. Cancer Cell 21: 309-322, 2012.

48. Zhu X, Wang K, Zhang K, Xu F, Yin Y, Zhu L and Zhou F: Galectin-1 knockdown in carcinoma-associated fibroblasts inhibits migration and invasion of human MDA-MB-231 breast cancer cells by modulating MMP-9 expression. Acta Biochim Biophys Sin (Shanghai) 48: 462-467, 2016.

49. Labernadie A, Kato T, Brugués A, Serra-Picamal X, Derzsi S, Arwert E, Weston A, González-Tarragó V, Elosegui-Artola A, Albertazzi L, et al: A mechanically active heterotypic E-cadherin/N-cadherin adhesion enables fibroblasts to drive cancer cell invasion. Nat Cell Biol 19: 224-237, 2017. 